\title{
Microecological investigation and comparison of two clinical methods to evaluate axillary osmidrosis
}

\author{
HONGJIAO DU, SHU DING, LIHUA GAO, JINRONG ZENG and JIANYUN LU
}

Department of Dermatology, The Third Xiangya Hospital, Central South University, Changsha, Hunan 410013, P.R. China

Received January 29, 2020; Accepted August 25, 2020

DOI: $10.3892 / \mathrm{mmr} .2020 .11528$

\begin{abstract}
Axillary osmidrosis (AO) is a common disease that causes patients to develop malodor and occurs worldwide. There is a lack of uniform standards to evaluate the severity of the odor and identify a sensitive and convenient method to determine the therapeutic effect of AO treatments in a clinical setting. In the present study, the association between $\mathrm{pH}$ value and disease severity was investigated and the potential pathogenic bacteria and probiotic pathogens of $\mathrm{AO}$ were further examined. A total of 32 patients with bilateral AO and 32 normal healthy controls were recruited for the present study. The odor was investigated using the traditional method (TM) and our groups newly developed $\mathrm{Lu}$ swab method (LSM) and according to the results, the cases were assigned a score on a 4-point scale. The patients' scores and $\mathrm{pH}$ value were recorded. The microbiological compositions of the affected sites were determined using 16S rDNA sequencing. The mean LSM score was higher compared with the mean TM score $(\mathrm{P}<0.05)$. Furthermore, the mean axillary $\mathrm{pH}$ value was higher in patients with $\mathrm{AO}$ compared with that in healthy subjects $(\mathrm{P}<0.0001)$, and the mean $\mathrm{pH}$ value of patients with high disease severity was higher compared with that in patients with moderate disease severity $(\mathrm{P}<0.001)$. In the microecological flora, the proportion of Staphylococcus species on patients with AO was significantly lower compared with that on normal controls $(\mathrm{P}<0.0001)$, while the proportion of Corynebacterium and Anaerococcus was significantly higher compared with that on normal controls $(\mathrm{P}<0.01$ and $\mathrm{P}<0.001$, respectively). In conclusion, LSM provided a higher sensitivity for evaluating odor severity than the TM and may be suitable for use in a clinical setting. The $\mathrm{pH}$ value was positively associated
\end{abstract}

Correspondence to: Professor Jianyun Lu or Dr Jinrong Zeng, Department of Dermatology, The Third Xiangya Hospital, Central South University, 138 Tongzipo Road, Yuelu, Changsha, Hunan 410013, P.R. China

E-mail: xiaoyun3@csu.edu.cn

E-mail: 1203534510@qq.com

Key words: axillary osmidrosis, microbiome, cotton swab method, $\mathrm{pH}$ value with AO severity. Staphylococcus may be an appropriate probiotic for the treatment of $\mathrm{AO}$, while Corynebacterium and Anaerococcus may be causative pathogens of AO. The present study was registered in the Chinese Clinical Trial Registry (registration no. ChiCTR2000037275).

\section{Introduction}

Axillary osmidrosis (AO) is a distressing disease that has a detrimental effect on the social lives of affected patients, due to the associated unpleasant odor and yellowish staining of clothing, which is more common in Asian countries (1). Odors may be produced by bacteria that colonize the initially odorless secretions of sweat and sebaceous glands (2), or it may be related to the expression level of apolipoprotein D (ApoD) in the apocrine sweat glands $(3,4)$. There are currently no standardized evidence-based clinical evaluation methods for AO. Traditional measures, such as those reported by Jung et al (5) and Wang et al (6), have been used in a clinical setting to evaluate the degree of malodor. However, traditional methods have a judgement bias and lack sensitivity (7). To solve these problems, the Lu swab method (LSM) was proposed by our laboratory, which has a higher sensitivity in determining the odor degree of AO by clinicians.

It is well-known that the composition and activity of the microbial community of the human axilla has a key role in the formation of body odor (8). Microbiological studies based on traditional culture have revealed that the skin-resident microbiota primarily consists of Gram-positive bacteria, such as Staphylococcus and Corynebacterium (2). These techniques form a sound basis; however, they misrepresent the true bacterial diversity of a complex community (9). The recent emerging 16S rDNA sequencing technology provides researchers with the opportunity to investigate the composition and the skin microbiome in a high-throughput manner (10). In addition, bacterial growth on the skin is pH-dependent (11). The skin's $\mathrm{pH}$ is normally acidic, with values ranging from 4 to 6. The physiological role of an acidic skin surface is to act as a defense barrier against invading organisms (12).

The present study aimed to identify a sensitive and convenient method to determine the therapeutic effect of $\mathrm{AO}$ in a clinical setting, to investigate the association between $\mathrm{pH}$ value and disease severity and to further examine the potential pathogenic bacteria and probiotic pathogens of AO. 


\section{Materials and methods}

Subjects. The present study was approved by the institutional review board of the Third Xiangya Hospital, Central South University (Hunan, China). All patients provided written informed consent. For participants below the age of 18 years, the legal guardians provided written consent. There was no compensation provided for participation. A total of 32 patients (64 axillae) had bilateral axillary osmidrosis (13). The exclusion criteria were as follows: i) Any treatment received in the previous 2 weeks, a serious infection or other disruptive skin disease in the axillary fossa; ii) current pregnancy or breastfeeding; and iii) severe systemic diseases. A total of 32 patients ( 24 females, 8 males; age range, $11-45$ years; mean age, $25.75 \pm 7.62$ years) and 32 healthy control subjects who had no axillary odor (17 females, 15 males; age range, $14-42$ years; mean age, $25.84 \pm 6.44$ years) with no history of dermatological disorders or other chronic medical disorders and with no current skin infections were enrolled at the Department of Dermatology, the Third Xiangya Hospital, Central South University between December 2017 and December 2018. The family history was primarily characterized by an unpleasant unilateral or bilateral smell in the armpits of the parents; 23 patients had a family history of the disease (Table I).

Clinical study design. The subjects were instructed not to use any products in the axillae, such as antiperspirants, talcum powder, perfume or bath additives, and to avoid eating spicy foods for 2 weeks prior to the test. Local cleansing was stopped following a shower taken on the evening prior to the test, and the test was performed in the morning. The test was performed in a comfortable temperature-controlled $\left(24-26^{\circ} \mathrm{C}\right)$ environment with closed doors and windows. After $15 \mathrm{~min}$ of daily activity (walk), the armpits of the subjects were exposed to rule out any interference from their clothes. A total of 2 designated physicians used the following two methods to simultaneously evaluate and determine the severity of AO for each patient, when they scored different levels, the mean scores were recorded. Each subject was examined three times. Furthermore, the physicians (JL and JZ) are trained to perform tests and they have ample experience and are involved in the development of tests in our laboratory.

Traditional method (TM). The physicians gradually approached the exposed armpit and rated the abnormal smell, according to the abnormal smell grading system, using the following scoring system: 0 , none; 1 , mild; 2 , moderate and 3 , severe (Table II) $(6,14)$.

LSM. Patients were instructed to fully expose their armpits and each side was wiped with cotton swabs 10 times by the physicians. The cotton swabs were then smelt immediately by two examiners while holding them 5-10 cm under the examiner's nose. According to the cotton swab odor degree grading system, the following scoring was used: 0 , none; 1 , mild; 2 , moderate; and 3, severe (Table III).

Measurement of $p H$. The $\mathrm{pH}$ value of the underarm skin surface was evaluated on the bilateral axilla following the olfactory assessment. In each patient, 3 measurements were obtained at the center of the axilla and the acquired average was used for subsequent calculations and statistical analysis. The in vivo skin surface $\mathrm{pH}$ value was measured non-invasively using a $\mathrm{PH} 30 \mathrm{~A} \mathrm{pH}$ meter (Clean), consisting of a combined flat glass probe and a reference electrode in a single probe. The $\mathrm{pH}$ meter was calibrated using standard buffers. The probe was rinsed with distilled water prior to each measurement.

Microbiome sampling. Sterile cotton swabs soaked with sterile saline were used to collect samples from the axillary sites of each patient with $\mathrm{AO}$ and each healthy subject. Skin samples were collected by rubbing the skin 10 times perpendicularly.

DNA extraction and sequencing. The DNA collected on the swabs was isolated by BGI Genomics and stored at $-80^{\circ} \mathrm{C}$ until further experimentation. The V4 region of the $16 \mathrm{~S}$ rDNA gene was amplified using the following primers to generate an amplicon library for each sample: Primer 515 forward, 5'-GTGCCAGCMGCCGCGGTAA-3' and primer 806 reverse, 5'-GGACTACHVGGGTWTCTAAT-3' (15). PCR amplification, cloning and sequencing were performed as previously described (16). The fragments in the DNA library, of the expected sizes, were selected using the Agencourt AMPure XP (Beckman Coulter, Inc.) according to the manufacturer's protocols and then subjected to qualification using a Bioanalyzer-2100 (Agilent Technologies, Inc.) and pair-end sequencing on a Hiseq-2500 (Illumina Inc.) (17).

Statistical analysis. Descriptive parameters, such as the mean and standard deviation for normally distributed continuous data and the frequencies and percentages for categorical data were calculated. Student's t-test was used to evaluate continuous variables and 95\% CIs were compared between the 2 groups. For multiple comparisons, one-way ANOVA was used to analyze differences among groups. Tukey's method was applied for the post-hoc test. All tests were 2-tailed and $\mathrm{P}<0.05$ was considered to indicate a statistically significant difference. SPSS version 21.0 (IBM Corp.) was used to perform the statistical analyses.

\section{Results}

Comparison of scoring methods to evaluate axillary odor. The TM was used to evaluate the degree of odor and 14 patients were graded as having mild, 8 as moderate and 10 as severe axillary odor. The mean TM score was $1.88 \pm 0.87$. When the LSM was used to evaluate these patients, 3 were graded as having mild, 16 as moderate and 13 as severe axillary odor, with a mean LSM score of $2.31 \pm 0.64$. The difference between the TM and LSM scores was statistically significant $(\mathrm{P}=0.03$; Fig. 1), demonstrating that the LSM was more sensitive compared with the TM in evaluating odor severity in patients with AO.

Comparison of axillary $\mathrm{pH}$. Comparison of the $\mathrm{pH}$ values of the left and right axillae in patients with $\mathrm{AO}$ revealed no significant difference between sites $(6.39 \pm 0.54$ vs. $6.47 \pm 0.59$; $\mathrm{P}>0.05$ ). Similarly, the $\mathrm{pH}$ value did not significantly differ between sites in the healthy volunteers $(5.80 \pm 0.31$ vs. $5.88 \pm 0.38$; $\mathrm{P}>0.05$; Fig. $2 \mathrm{~A}$ ). The $\mathrm{pH}$ value of the ipsilateral 
Table I. Clinical information of the patients with axillary osmidrosis and healthy volunteers.

\begin{tabular}{|c|c|c|c|}
\hline Characteristic & Patients $(n=32)$ & Healthy subjects $(n=32)$ & P-value \\
\hline Age (years) & $25.75 \pm 7.62$ & $25.84 \pm 6.44$ & 0.9578 \\
\hline $\operatorname{Sex}, \mathrm{n}(\%)$ & & & 0.068 \\
\hline Male & $8(25)$ & $15(46.88)$ & \\
\hline Female & $24(75)$ & $17(53.13)$ & \\
\hline Family history, n (\%) & & & l \\
\hline Yes & $23(71.88)$ & $0(0)$ & \\
\hline No & $9(28.13)$ & $32(100)$ & \\
\hline Disease course (years) & $10.22 \pm 7.14$ & 0 & / \\
\hline
\end{tabular}

Values are expressed as $\mathrm{n}$ or the mean \pm standard deviation.

Table II. Axillae odor analogue scale.

\begin{tabular}{lll}
\hline Score & Degree & \multicolumn{1}{c}{ Definition } \\
\hline 0 & None & No odor detected within $5 \mathrm{~cm}$ \\
1 & Mild & Odor detected within $15 \mathrm{~cm}$ \\
2 & Moderate & Odor detected within $15-30 \mathrm{~cm}$ \\
3 & Severe & Odor detected $>30 \mathrm{~cm}$ \\
\hline
\end{tabular}

Table III. Grading scores of odor degree of the cotton swabs.

\begin{tabular}{lll}
\hline Score & Degree & \multicolumn{1}{c}{ Definition } \\
\hline 0 & None & No odor detected \\
1 & Mild & Faint peculiar odor \\
2 & Moderate & Obvious peculiar odor \\
3 & Severe & Pungent odor \\
\hline
\end{tabular}

axilla in patients with $\mathrm{AO}$ was significantly higher compared with that in healthy volunteers $(\mathrm{P}<0.0001$; Fig. $2 \mathrm{~A})$. This result indicated that an increased $\mathrm{pH}$ in the armpit may be associated with the onset of $\mathrm{AO}$.

The $\mathrm{pH}$ values of the axillae in patients with different degrees of malodor were also compared and the results indicated that the axillary $\mathrm{pH}$ value in patients graded as having moderate odor was significantly lower compared with that in patients graded as having severe odor $(6.23 \pm 0.29$ vs. $6.77 \pm 0.70$; $\mathrm{P}<0.001$; Fig. 2B). The $\mathrm{pH}$ value of the axillae in patients graded as having mild odor was also significantly lower compared with that in patients graded as having severe odor (6.05 \pm 0.07 vs. $6.77 \pm 0.70 ; \mathrm{P}<0.0001$; Fig. $2 \mathrm{~B})$. This suggests that a more severe condition of the patient was associated with a higher $\mathrm{pH}$ of the armpit.

Axillary microecology. As presented in Fig. 3A, there were genus-level changes in the composition of the microbial community in the case group. The axillary flora was randomly detected in 10 healthy volunteers and 10 patients

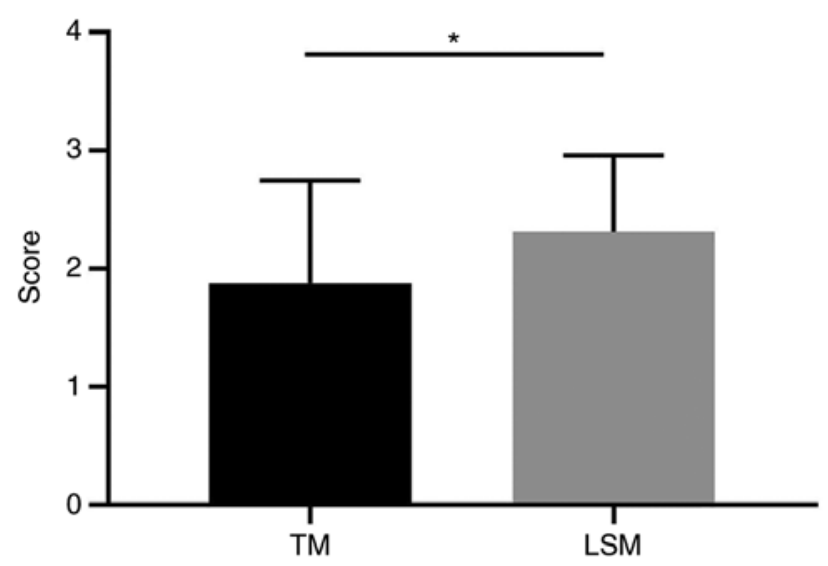

Figure 1. Comparison of the TM and the LSM for evaluating odor severity in patients with axillary osmidrosis. ${ }^{*} \mathrm{P}<0.05$. TM, traditional method; LSM, Lu swab method.

with moderate to severe AO. The major bacteria in the control group were Staphylococcus and Corynebacterium. Staphylococcus species accounted for a large proportion of the bacteria in the armpits (range, 54.42-91.94\%; mean, $71.64 \%$ ), while the second and third most common bacteria were Corynebacterium (16.26\%) and Anaerococcus $(2.20 \%)$, respectively. By contrast, the dominant genus in the malodor group was Corynebacterium and Anaerococcus (43.94 and $20.17 \%$, respectively). Of note, the proportion of Staphylococcus species was reduced (23.01\%). These results indicated that AO may be associated with the elevated ratios of Corynebacterium and Anaerococcus. As the proportion of Staphylococcus species was decreased in AO, it may be a useful probiotic for treating $\mathrm{AO}$.

Quantitative comparison of the major flora between patients and healthy subjects revealed that Staphylococcus was less prevalent in patients compared with that in healthy subjects, with an average reduction of $48.63 \%(23.01 \pm 18.45$ vs. $71.64 \pm 11.89 \%$; $\mathrm{P}<0.0001)$, whereas the proportion of Corynebacterium species was significantly higher in patients compared with that in the healthy subjects with an average increase of $27.68 \%$ (43.94 \pm 22.46 vs. $16.26 \pm 9.38$; $\mathrm{P}<0.01)$. In addition, the ratio of Anaerococcus species was higher in 
A

A

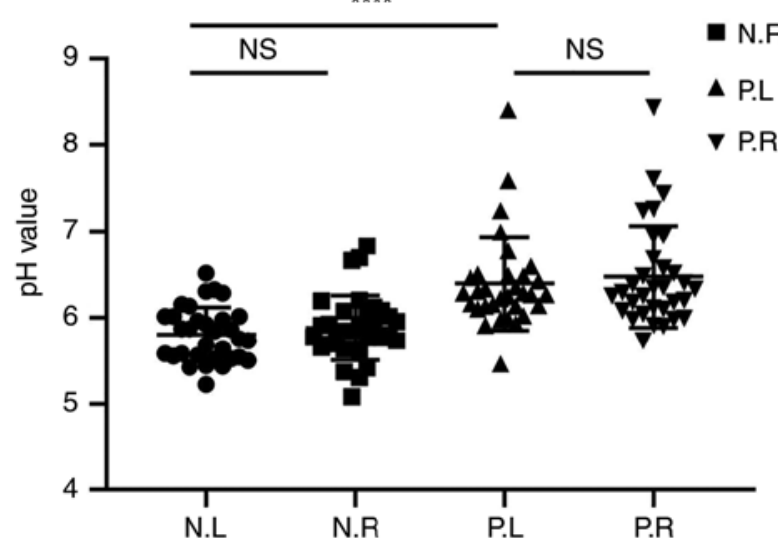

B

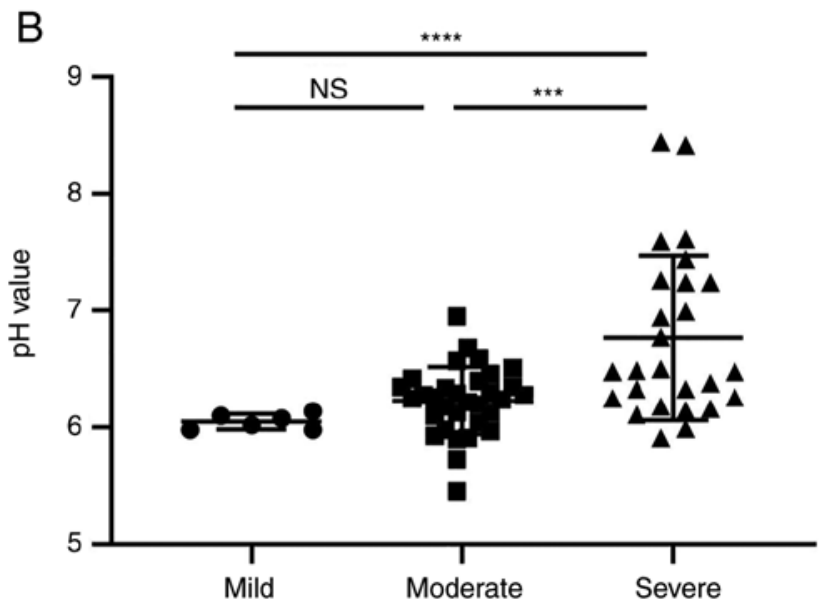

Figure 2. (A) $\mathrm{pH}$ value in patients with $\mathrm{AO}$ and healthy subjects. (B) Comparison of $\mathrm{pH}$ values in patients with different degrees of $\mathrm{AO} .{ }^{* * * *} \mathrm{P}<0.001 ;{ }^{* * * * *} \mathrm{P}<0.0001$. N.L, normal left; N.R, normal right; P.L, patient left; P.R, patient right; NS, not significant; AO, axillary osmidrosis.

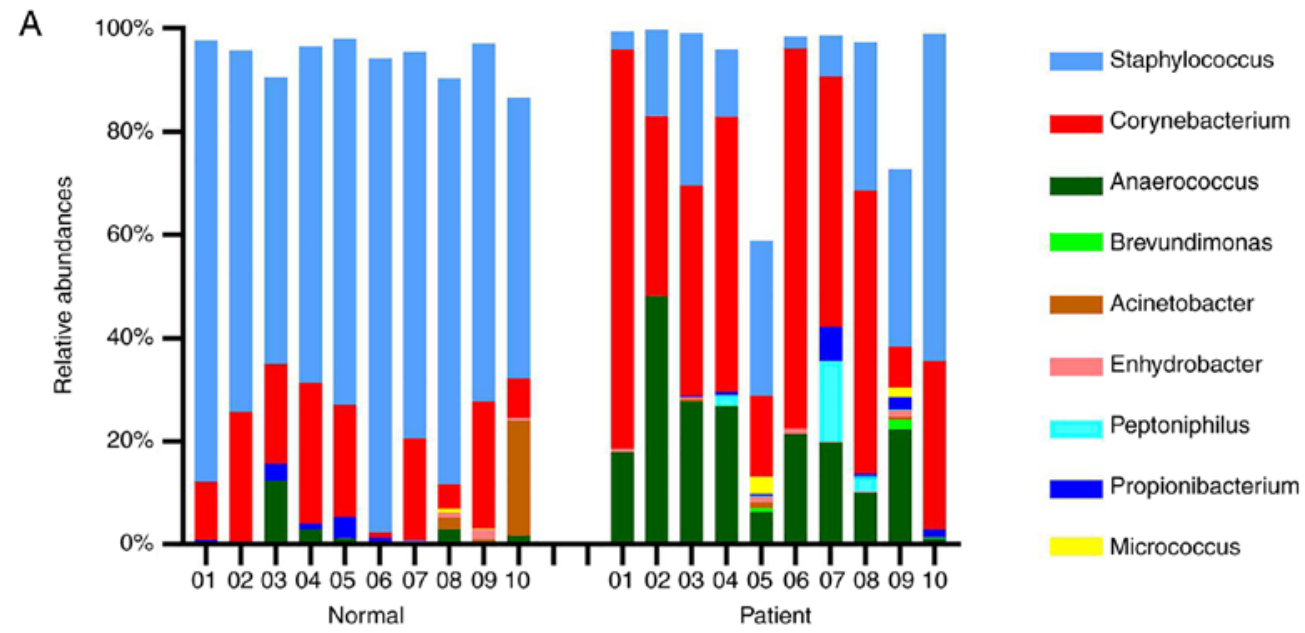

B

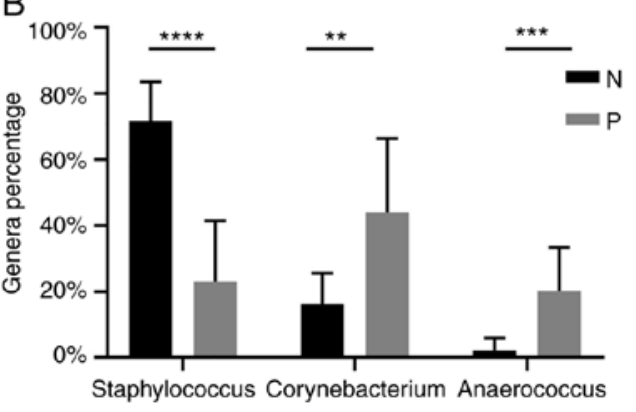

C

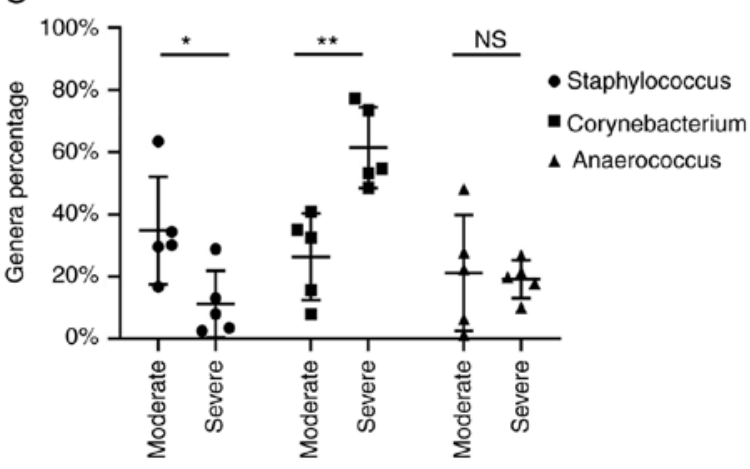

Figure 3. (A) Microbial composition in the armpits of healthy subjects and patients with AO. (B) Comparison of the main flora in normal subjects and patients with AO. (C) Comparison of bacterial populations by odor degree in patients with $\mathrm{AO} .{ }^{*} \mathrm{P}<0.05 ;{ }^{* *} \mathrm{P}<0.01 ;{ }^{* * * *} \mathrm{P}<0.001 ;{ }^{* * * * *} \mathrm{P}<0.0001$. NS, not significant; $\mathrm{N}$, normal; P, patient; $\mathrm{AO}$, axillary osmidrosis.

patients with AO compared with that in healthy volunteers, with an average increase of $17.97 \%$ (20.17 \pm 13.13 vs. $2.20 \pm 3.72$; $\mathrm{P}<0.001$; Fig. 3B). These results suggested that Anaerococcus and Corynebacterium species may be accountable for the production of the strong odor in patients with $\mathrm{AO}$.

Furthermore, the main flora differences in patients with various disease severities were also compared (Fig. 3C). The amount of Corynebacterium species was higher in patients with severe disease compared with that in those with moderate disease $(61.51 \pm 12.99$ vs. $26.37 \pm 13.94 ; \mathrm{P}<0.01)$, indicating that Corynebacterium may also be a strong odor-secreting microbe (18). There was no significant difference in the population of Anaerococcus between the moderate and severe odor groups. The proportion of the Staphylococcus species exhibited the opposite trend, as it was reduced in patients with severe odor as compared with that in patients 
with moderate odor $(11.17 \pm 10.72$ vs. $34.84 \pm 17.33 ; \mathrm{P}<0.05)$, suggesting that the proportion may be negatively associated with the odor degree.

\section{Discussion}

In the present study, the LSM was used to determine odor severity. According to our general experience, using the TM, moderate odor may be easily misjudged as mild, while the LSM was more sensitive to distinguish mild from moderate odors compared with TM. Therefore, it was concluded that LSM was comparatively more sensitive to evaluate the severity of $\mathrm{AO}$, and it was more convenient to use in clinical practice.

The $\mathrm{pH}$ of the skin surface may also influence the cutaneous microflora (19). Impaired acidification of the axillary $\mathrm{pH}$ in patients may be a possible factor for promoting host susceptibility to pathogenic bacteria. A previous study revealed that patients with diabetes had a significantly higher axillary $\mathrm{pH}$ level compared with that in healthy controls and candida infection in patients with diabetes was also associated with the elevated $\mathrm{pH}$ value in their armpits (20). Another study identified an association between a high skin $\mathrm{pH}$ and the growth of microorganisms that produce malodor (21). In the present study, the $\mathrm{pH}$ value in the axillary fossa of the patients was significantly higher compared with that in normal controls, which may be associated with the bacteria that produce odor. The difference between the $\mathrm{pH}$ values of the right and left armpit was not statistically significant in either group, which was consistent with previous studies $(22,23)$. In addition, it was observed that the different odor severities were significantly associated with different $\mathrm{pH}$ values of the axilla: The $\mathrm{pH}$ value in patients with severe $\mathrm{AO}$ was significantly higher compared with that in patients with mild and moderate $\mathrm{AO}$, indicating that a higher axillary $\mathrm{pH}$ value was associated with the production of a stronger odor.

The production of malodor on the underarm skin is primarily attributed to the action of the resident microbiota on odorless natural secretions from the apocrine gland (24). Corynebacterium is one of the dominant bacterial groups in the armpit microbiota, which has an important role in malodor generation (25). Furthermore, a previous study revealed that the Anaerococcus species in sweat contributed to axillary odor (26). Recently, an analysis using next-generation sequencing (without culturing) confirmed that the skin microbiota contains Anaerococcus $(2,27)$. In the present study, the proportions of Anaerococcus and Corynebacterium species in patients with axillary osmidrosis were higher compared with those in healthy subjects, indicating that Anaerococcus and Corynebacterium species may contribute to the production of odor. It was also indicated that different odor severities were associated with the axillary microbiome composition. Corynebacterium species were significantly elevated in patients with severe $\mathrm{AO}$ compared with those in patients with moderate $\mathrm{AO}$, indicating that Corynebacterium was associated with body odor. The results are also consistent with the data from a culture-based study (18). On the contrary, Staphylococcus species were significantly more prevalent in patients with moderate AO compared with those in patients with severe AO, in normal subjects, the Staphylococcus concentration was highest and that they are the major constituent of a normal armpit flora, indicating that the Staphylococcus species may be negatively associated with odor strength and may serve as a potential probiotic. Okamoto et al (28) determined that at the genus level, there was no direct association between Staphylococcus species counts and malodor intensity in the axilla; however, this may not preclude a role of these microorganisms in the production of odorant There was no significant difference in the proportion of Anaerococcus between patients with moderate disease and those with severe disease. The possible reasons for this include the following: i) Anaerococcus grows slowly, so no significant difference may be observed over a short period of time $(29,30)$; and ii) the insufficient sample size was unable to reduce variances due to individual variations in Anaerococcus susceptibility to environmental factors; and iii) the characteristics of their sensitivity to oxygen (29).

The technological limitations of 16S rDNA sequencing allow it to identify only a small proportion of strains at the species level. Most bacteria may only be authenticated at the genus level. Furthermore, the small number of physicians performing the smell scoring may be a limitation of the present study. Additional further exploratory research is urgently required to increase the understanding of the association between the microbiome and odor generation.

In conclusion, the present study demonstrated that the LSM was more sensitive in detecting odor severity and more convenient for use in the clinical setting than the TM. The axillary $\mathrm{pH}$ value of patients with $\mathrm{AO}$ was higher compared with that in healthy subjects, and a more severe the condition of $\mathrm{AO}$ was associated with a higher the $\mathrm{pH}$ value in the armpit. Corynebacterium and Anaerococcus may be pathogenic bacteria of AO, while the role of Staphylococcus species as a potential probiotic requires confirmation in further clinical trials.

\section{Acknowledgements}

Not applicable.

\section{Funding}

The current study was funded by New Xiangya Talent Project of the Third Xiangya Hospital of Central South University (grant no. 20170309 to JL).

\section{Availability of data and materials}

The datasets generated and/or analyzed during the current study are available from the SRA repository (https://www. ncbi.nlm.nih.gov/sra/?term=PRJNA646090) under the accession number PRJNA646090.

\section{Authors' contributions}

JL conceived and designed the present study. JL, JZ and HD performed the experiments. JZ and SD analyzed the data and prepared the images. LG analyzed the data and discussed the results. HD and LG drafted the manuscript. All authors read and approved the final manuscript. 


\section{Ethics approval and consent to participate}

The present study was approved by the institutional review board of the Third Xiangya Hospital, Central South University (Hunan, China). All patients provided written informed consent. For participants below the age of 18 years, the legal guardians provided written consent.

\section{Patient consent for publication}

Not applicable.

\section{Competing interests}

The authors declare that they have no competing interests.

\section{References}

1. Toyoda $\mathrm{Y}$, Gomi T, Nakagawa $\mathrm{H}$, Nagakura $\mathrm{M}$ and Ishikawa $\mathrm{T}$ Diagnosis of human axillary osmidrosis by genotyping of the human ABCC11 gene: Clinical practice and basic scientific evidence. Biomed Res Int 2016: 7670483, 2016.

2. James AG, Austin CJ, Cox DS, Taylor D and Calvert R: Microbiological and biochemical origins of human axillary odour. FEMS Microbiol Ecol 83: 527-540, 2013.

3. Wood A and Kelly D: Skin microbiology, body odor, and methylotrophic bacteria. Handbook Hydrocarbon Lipid Microbiol 2010 3204-3213, 2010.

4. Chen H, Yang G, Li Y, Li X and Du J: Expression of apolipoprotein $\mathrm{D}$ and androgen receptor in axillary osmidrosis and its molecular mechanism. Int J Clin Exp Med 6: 497-503, 2013.

5. Jung SW, Lee SJ and Park HR: Comparison of outcomes of two methods of axillary osmidrosis surgery: Subdermal excision versus liposuction combined with diode laser ablation. Arch Aesthetic Plast Surg 26: 20-27, 2020.

6. Wang R, Yang J and Sun J: A minimally invasive procedure for axillary osmidrosis: Subcutaneous curettage combined with trimming through a small incision. Aesthetic Plast Surg 39: 106-113, 2015.

7. Memarian F, Amani-Tehran M and Latifi M: Rank ordering and image processing methods aided fabric wrinkle evaluation. Fibers Polym 12: 830, 2011.

8. Egert M, Höhne HM, Weber T, Simmering R, Banowski B and Breves R: Identification of compounds inhibiting the C-S lyase activity of a cell extract from a Staphylococcus sp. isolated from human skin. Lett Appl Microbiol 57: 534-539, 2013.

9. Gao Z, Tseng CH, Pei Z and Blaser MJ: Molecular analysis of human forearm superficial skin bacterial biota. Proc Natl Acad Sci USA 104: 2927-2932, 2007.

10. Fredrich E, Barzantny H, Brune I and Tauch A: Daily battle against body odor: Towards the activity of the axillary microbiota. Trends Microbiol 21: 305-312, 2013.

11. Tarun J, Susan J, Suria J, Susan VJ and Criton S: Evaluation of $\mathrm{pH}$ of bathing soaps and shampoos for skin and hair care. Indian J Dermatol 59: 442-444, 2014.

12. Ali SM and Yosipovitch G: Skin pH: From basic science to basic skin care. Acta Derm Venereol 93: 261-267, 2013.

13. Dai Y, Xu AE and He J: A refined surgical treatment modality for bromhidrosis: Subcutaneous scissor with micropore. Dermatol Ther 30: 2017.

14. He J, Wang T and Dong J: A close positive correlation between malodor and sweating as a marker for the treatment of axillary bromhidrosis with botulinum toxin A. J Dermatolog Treat 23: 461-464, 2012.
15. Ring HC, Thorsen J, Saunte DM, Lilje B, Bay L, Riis PT, Larsen N, Andersen LO, Nielsen HV, Miller IM, et al: The follicular skin microbiome in patients with hidradenitis suppurativa and healthy controls. JAMA Dermatol 153: 897-905, 2017.

16. Grice EA, Kong HH, Conlan S, Deming CB, Davis J, Young AC; NISC Comparative Sequencing Program, Bouffard GG, Blakesley RW, Murray PR, et al: Topographical and temporal diversity of the human skin microbiome. Science 324: 1190-1192, 2009.

17. Fadrosh DW, Ma B, Gajer P, Sengamalay N, Ott S, Brotman RM and Ravel J: An improved dual-indexing approach for multiplexed $16 \mathrm{~S}$ rRNA gene sequencing on the Illumina MiSeq platform. Microbiome 2: 1-6, 2014.

18. Troccaz M, Gaia N, Beccucci S, Schrenzel J, Cayeux I, Starkenmann C and Lazarevic V: Mapping axillary microbiota responsible for body odours using a culture-independent approach. Microbiome 3: 3, 2015.

19. Rippke F, Berardesca $\mathrm{E}$ and Weber TM: $\mathrm{pH}$ and microbial infections. Curr Probl Dermatol 54: 87-94, 2018.

20. Behm B, Schreml S, Landthaler M and Babilas P: Skin signs in diabetes mellitus. Eur Acad Dermatol Venereol 26: 1203-1211, 2012.

21. Kemper M, Bielfeldt S, Knie U, Wilhelm KP and Abels C: Significant reduction of body odor in older people with a $\mathrm{pH} 4.0$ emulsion. Cosmetics 2: 136-145, 2015.

22. Williams S, Davids M, Reuther T, Kraus D and Kerscher M: Gender difference of in vivo skin surface $\mathrm{pH}$ in the axilla and the effect of a standardized washing procedure with tap water. Skin Pharmacol Physiol 18: 247-252, 2005.

23. Stenzaly-Achtert S, Schölermann A, Schreiber J, Diec KH, Rippke $\mathrm{F}$ and Bielfeldt $\mathrm{S}$ : Axillary $\mathrm{pH}$ and influence of deodorants. Skin Res Technol 6: 87-91, 2008.

24. Ren Y, Liu W, Chen J, Wang J, Wang K, Zhou J, Cai S, Zheng M, Liu J, Liu L and Xue D: A missense variant of the ABCC11 gene is associated with axillary osmidrosis susceptibility and clinical phenotypes in the Chinese han population. Sci Rep 7: 46335, 2017.

25. Li M, Budding AE, van der Lugt-Degen M, Du-Thumm L, Vandeven $\mathrm{M}$ and Fan A: The influence of age, gender and race/ethnicity on the composition of the human axillary microbiome. Int J Cosmet Sci 41: 371-377, 2019.

26. Fujii T, Shinozaki J, Kajiura T, Iwasaki K and Fudou R: A newly discovered Anaerococcus strain responsible for axillary odor and a new axillary odor inhibitor, pentagalloyl glucose. FEMS Microbiol Ecol 89: 198-207, 2014.

27. Minhas GS, Bawdon D, Herman R, Rudden M, Stone AP, James AG, Thomas GH and Newstead S: Structural basis of malodour precursor transport in the human axilla. Elife 7 : e34995, 2018

28. Okamoto H, Koizumi S, Shimizu H, Cho O and Sugita T: Characterization of the axillary microbiota of Japanese male subjects with spicy and milky odor types by pyrosequencing. Biocontrol Sci 23: 1-5, 2018.

29. Murphy EC and Frick IM: Gram-positive anaerobic cocci-commensals and opportunistic pathogens. FEMS Microbiol Rev 37: 520-553, 2013.

30. Badri M, Nilson B, Ragnarsson S, Senneby E and Rasmussen M: Clinical and microbiological features of bacteraemia with gram-positive anaerobic cocci: A population-based retrospective study. Clin Microbiol Infect 25: 760.e1-760.e6, 2019.

This work is licensed under a Creative Commons Attribution-NonCommercial-NoDerivatives 4.0 International (CC BY-NC-ND 4.0) License. 